\title{
Phage or foe: an insight into the impact of viral predation on microbial communities
}

\author{
Lucía Fernández ${ }^{1}$ - Ana Rodríguez ${ }^{1}$ Pilar García ${ }^{1}$
}

Received: 29 August 2017 / Revised: 24 October 2017 / Accepted: 21 December 2017 / Published online: 25 January 2018

(c) International Society for Microbial Ecology 2018

\begin{abstract}
Since their discovery, bacteriophages have been traditionally regarded as the natural enemies of bacteria. However, recent advances in molecular biology techniques, especially data from "omics" analyses, have revealed that the interplay between bacterial viruses and their hosts is far more intricate than initially thought. On the one hand, we have become more aware of the impact of viral predation on the composition and genetic makeup of microbial communities thanks to genomic and metagenomic approaches. Moreover, data obtained from transcriptomic, proteomic, and metabolomic studies have shown that responses to phage predation are complex and diverse, varying greatly depending on the bacterial host, phage, and multiplicity of infection. Interestingly, phage exposure may alter different phenotypes, including virulence and biofilm formation. The complexity of the interactions between microbes and their viral predators is also evidenced by the link between quorum-sensing signaling pathways and bacteriophage resistance. Overall, new data increasingly suggests that both temperate and virulent phages have a positive effect on the evolution and adaptation of microbial populations. From this perspective, further research is still necessary to fully understand the interactions between phage and host under conditions that allow co-existence of both populations, reflecting more accurately the dynamics in natural microbial communities.
\end{abstract}

\section{One hundred years of phage history}

A century ago, two independent studies [1,2] reported the identification of a novel bacteriolytic agent: the bacteriophage. In the context of the pre-antibiotic era, when infectious diseases were decimating the population, the discovery of a microbe with potential to fight bacterial pathogens was definitely welcome. Nevertheless, phage therapy was soon overshadowed by the introduction of antibiotics, which were easier to use and exhibited a wider spectrum of action. Nowadays, antibiotic misuse and abuse have led to an explosion in bacterial resistance. This current landscape has been conducive to a renewed interest in phage therapy, but considering the advances in molecular biology and our greater understanding of bacteriophages [3]. Phages are also increasingly recognized as an integral part of environmental communities and microbiota [4, 5].

Lucía Fernández

lucia.Fernandez@ipla.csic.es

1 Instituto de Productos Lácteos de Asturias (IPLA-CSIC), Paseo Río Linares s/n, 33300 Villaviciosa, Asturias, Spain
Despite a declining interest in phage therapy throughout most of the 20th century, bacteriophages have retained a prominent place in scientific research. Indeed, phage research was a key factor for the development of genetic engineering tools [6]. Recently, the discovery of CRISPR-Cas systems revealed the ability of microbes to acquire adaptive immunity against bacteriophages [7]. Shortly afterwards, CRISPR-Cas systems came to the spotlight as sophisticated genome editing tools [8]. All this information clearly demonstrates the positive impact of bacteriophages on human applications. However, the interactions between phages and their hosts in natural environments are just starting to be unveiled. In this context, temperate phages have been more widely considered potentially beneficial for their host, while lytic phages have been mostly regarded as predators with antimicrobial potential. However, recent findings suggest that lytic phages may play a similar role in bacterial communities to that of temperate phages, but differ in the mechanisms that avoid complete eradication of their host. This is partly accomplished through a tight coordination between bacterial population development and phage resistance.

This review intends to compile, dissect, and discuss the current knowledge about the interplay between 
Fig. 1 Examples of bacterial responses to phage exposure identified with different "omics" analyses. The drawing depicts a bacterial cell showing the physiological processes that changed upon phage predation. In most cases, there was a synchronized infection of the bacterial population with a virulent phage or induction of the lytic cycle in lysogenic bacteria. However, in the case of $S$. aureus, data represent differences between a biofilm subject to low-level predation with a lytic phage and a control biofilm

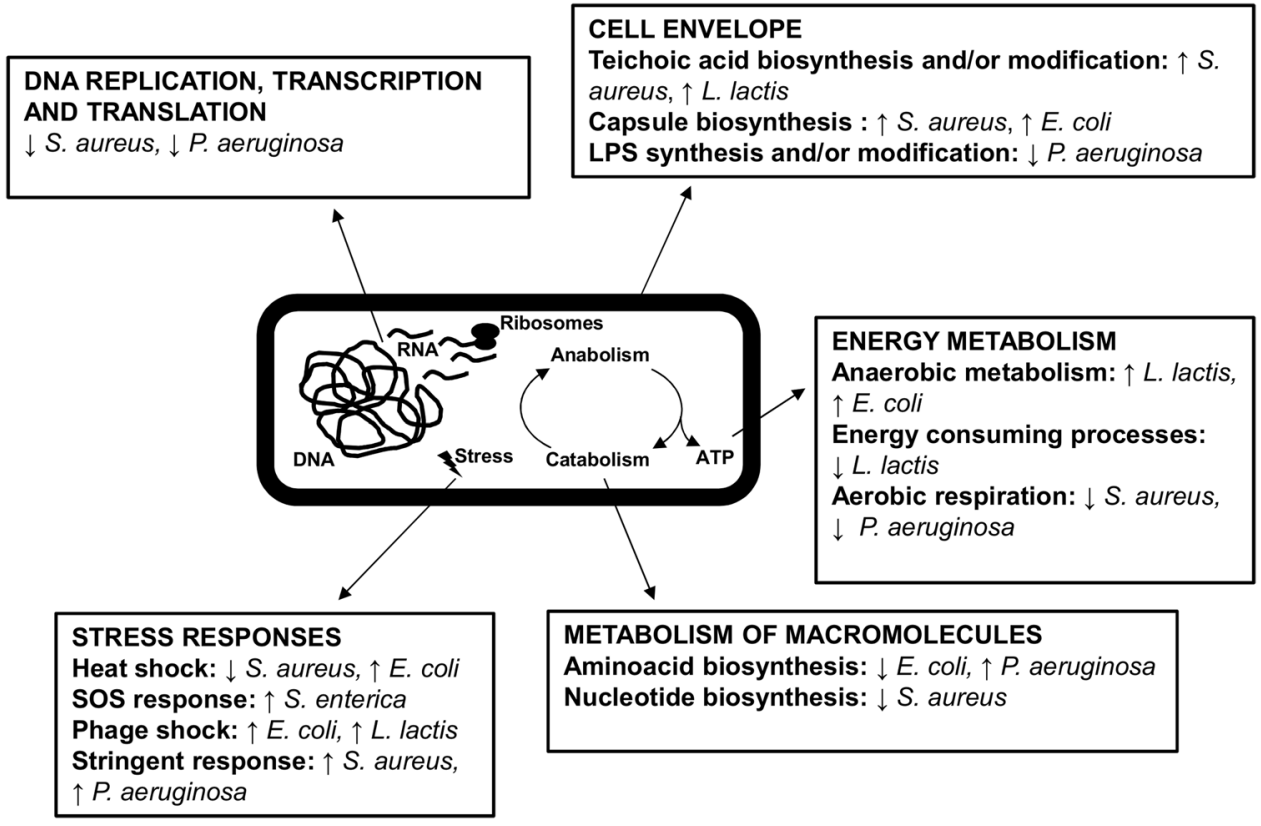

bacteriophages and microbial populations. To do that, we will examine how phages modulate the metagenome of bacterial communities, the impact of phage exposure on the transcriptome, proteome, and metabolome of the host, and how viral predation can alter different bacterial phenotypes. Last but not the least, we will discuss novel evidence showing that bacteria-phage interactions are under the control of cell-to-cell signaling. Taken together, this data hints that the presence of bacteriophages, both temperate and virulent, can exert a positive effect on the overall fitness of bacterial populations and highlights the need to conduct further research on this topic.

\section{Phage predation as an evolutionary force}

Bacteriophages play a major role in the evolution of microbial communities in natural and man-made environments alike. Indeed, genomic studies have revealed that phage genetic material may account for about $20 \%$ of some bacterial genomes [9]. Moreover, as stressors, phages can exert selective pressure and transform the composition of the community. A clear example is the selection of phageresistant mutants, which can display additional phenotypes such as virulence attenuation. For instance, phage-resistant strains of Yersinia pestis were less lethal in a mouse model [10] and displayed a loss of colonization fitness in Campylobacter jejuni [11]. Conversely, phage-resistant mutants of Pseudomonas aeruginosa displayed increased production of extracellular toxins and caused greater damage to mammalian cell lines [12]. Additionally, Davies et al. [13] observed that selective pressure exerted by temperate phages may accelerate adaptive evolution of bacterial pathogens during infection of the host. Thus, it appears that exposure to bacteriophages can enhance adaptability and/or virulence of bacteria, a possibility that should be examined when selecting candidates for phage therapy applications.

An interesting study recently showed that an increased mutation rate in bacteria may be deleterious for the predator bacteriophage population [14]. Thus, simultaneous exposure of Pseudomonas fluorescence to streptomycin and the lytic phage SBW25 2 resulted in a higher rate of phageresistant bacteria than exposure to the phage alone, leading to disappearance of the viral particles. This suggested that antibiotics may increase the mutation rate, perhaps by favoring mutator phenotypes [14]. More importantly, this result indicates that antibiotics can alter the natural coevolution of phage-host populations. This is concerning because coevolution between phage and prey is recognized as a major driving force of evolutionary processes that modulates diversity in microbial communities and, ultimately, affects ecological cycles [15].

Another mechanism involved in microbial evolution is horizontal gene transfer (HGT). Temperate phages are known mediators in this process [16], and have been related to the spread of virulence and/or resistance traits within bacterial communities. Haaber et al. [17] presented an autotransduction model that explains phage-mediated dissemination of antibiotic resistance determinants among Staphylococcus aureus strains. Worryingly, Modi et al. [18] observed increased prevalence of resistance gene markers in phage metagenomes in response to antibiotic use. 
Bacteriophages can also promote HGT through natural transformation. Keen et al. [19] described lytic phages called "superspreaders" that can release intact plasmid DNA into the surrounding milieu, probably because the genomes of these phages lack endonuclease-coding genes. These superspreader phages may contribute to spreading antibiotic resistance genes within natural communities and therefore should be avoided in phage therapy. Beyond that, superspreader phages reveal how virulent phages can also promote gene mobilization within bacterial populations.

\section{Bacterial responses to viral infection}

Besides changing the genetic makeup of microbial communities, bacteriophages may modulate the physiological state of bacteria without genome modifications. Analysis of these responses to phage predation has been facilitated by the arrival of the "omics" techniques (Fig. 1). Indeed, we now have data from transcriptomic, proteomic, and metabolomic analyses of microbes upon phage exposure. Interestingly, the most dramatic changes seem to occur at late infection stages, such as those of virion assembly and lytic enzyme production [20, 21]. Also, there is evidence that transcriptional responses vary depending on the specific bacterial strain [22] and the bacteriophage [23, 24]. Most studies to date have assessed the responses of bacterial cells following synchronized infection with virulent phages or after induction of the lytic cycle in lysogenic bacteria. This information helps to understand the molecular interactions between phage and host bacterial cell throughout the lytic cycle. However, such a scenario does not reflect the most common situation in nature, in which bacterial cells may be undergoing different infection stages while others remain uninfected. To our knowledge, only one study so far has examined how phage predation affects the bacterial population transcriptome during a non-synchronized infection [25]; therefore, further research remains necessary in this area. Moreover, techniques like single-cell transcriptomics could reveal differences between the transcriptomes of individual cells in a phage-infected population. In this section we will show how responses to phage predation affect diverse aspects of bacterial physiology in the infected cells, although this information needs to be complemented with data regarding the responses to phage predation of uninfected cells.

\section{Metabolism}

Viral infection involves a hostile take-over of the host's machinery. Unsurprisingly, most studies of bacteria-phage interactions indicate dramatic changes in the host cell metabolism. Regarding energy metabolism, the most widespread response is a downregulation of genes related to the energy status of cells [25-30]. Additionally, infection with lytic phages leads to an upregulation of anaerobic respiration genes in L. lactis and E. coli [20, 31]. Phage infection also alters the expression of genes involved in the metabolism of macromolecules [20, 25, 26, 28, 30, 31]. Interestingly, a recent metabolomics study revealed that only some metabolites changed similarly when $P$. aeruginosa was infected by different phages, while most metabolites displayed different trends depending on the virus [23]. This suggests that there is no general metabolic response to phage infection, but rather that specific molecular interactions are established depending on the phage and host bacterial strain. Another interesting effect on host cell metabolism is the direct inhibition of RNA processing by a protein (Dip) produced by the giant phage $\phi \mathrm{KZ}$, which binds to RNase E protecting viral RNA from degradation $[32,33]$. In $P$. aeruginosa, infection by phages PAK_P4 and PAK_P3 leads to a fast global depletion of host transcripts and upregulation of an operon involved in RNA processing [24, 34]. Of note, host gene shutoff has also been observed in plant and animal cells during viral infection [35].

\section{Cell envelope}

Some studies found that phage exposure affected transcription of genes involved in biosynthesis or modification of the cell envelope. For instance, L. lactis cells infected by the lytic phage $\mathrm{c} 2$ showed upregulation of genes required for the production of a teichoic acid precursor and for D-alanylation of cell wall teichoic acids [31]. In a similar manner, lytic infection by the temperate phage Tuc2009 induced a D-Ala-D-Ala carboxypeptidase that participates in peptidoglycan modification [21]. In contrast, Lavigne et al. [26] reported downregulation of the $w b p$ and arn operons, respectively, involved in LPS biosynthesis and lipid A modification, during phage infection of $P$. aeruginosa. In $E$. coli, Poranen et al. [20] observed upregulation of genes necessary for capsule synthesis at $10 \mathrm{~min}$ postinfection. $S$. aureus biofilm cells infected with the lytic phage phiIPLA-RODI displayed induction of capsule-related genes and genes necessary for D-Ala modification of teichoic acids, while genes involved in peptidoglycan biosynthesis were repressed [25]. Perhaps these changes to the cell surface have the role of protecting the infected bacteria from infection by other phages, but they could also have side-effects related to virulence and/or antimicrobial resistance. 


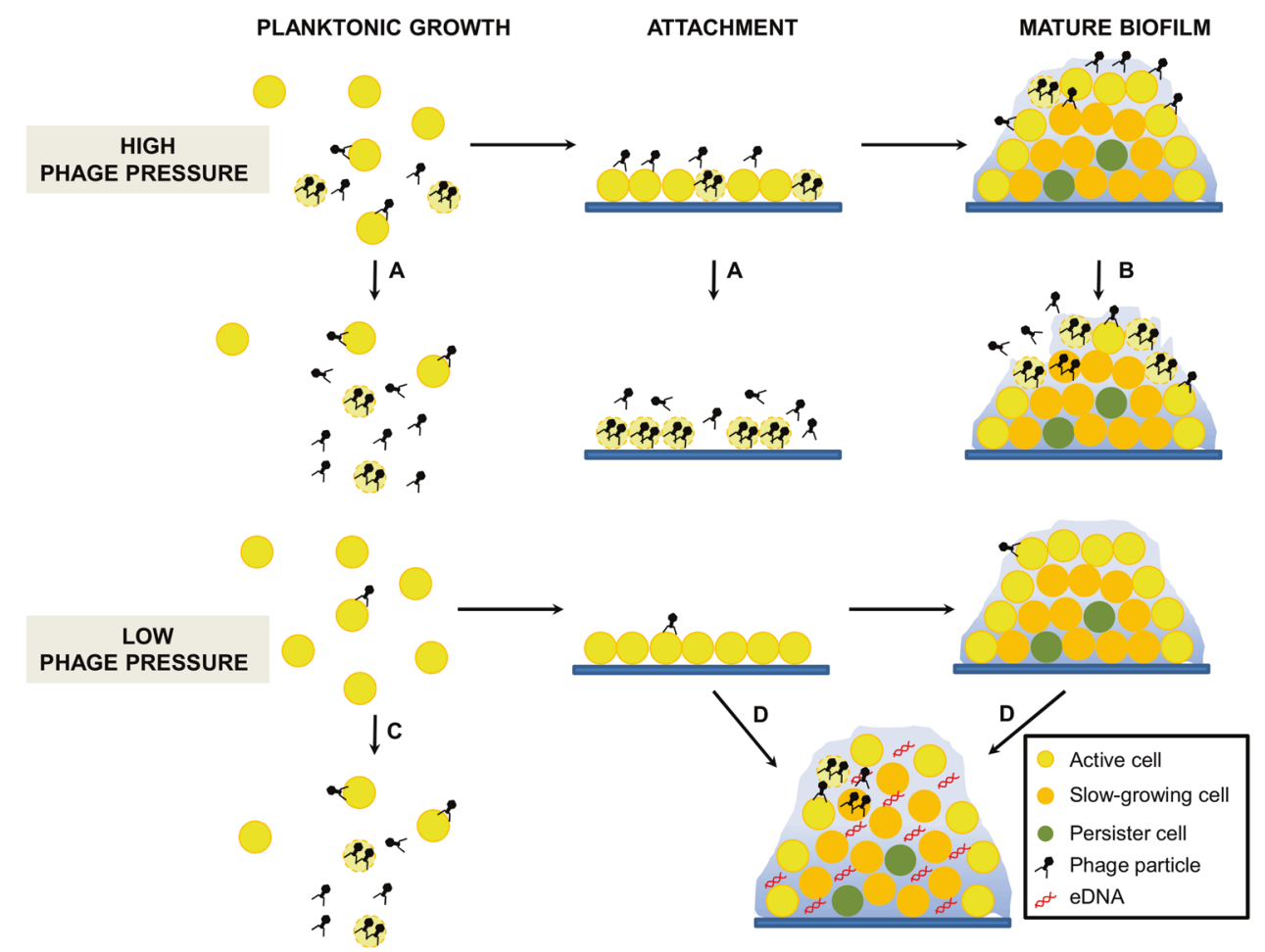

Fig. 2 Interplay between bacteriophages and bacterial biofilms. Cell aggregation may favor phage propagation in planktonic cultures or attached cells not surrounded by a matrix. (a) especially if phage pressure is high, resulting in complete eradication of the microbial population. However, arrangement of the cells in a mature biofilm will delay penetration of the viral particles and slow down the infection (b). When phage pressure is low, attachment to a surface may be the

\section{Stress responses}

Not surprisingly, phage infection can elicit diverse stress responses in the host. For example, lytic infection triggers the SOS response in Salmonella enterica ser. Typhimurium [36] and the heat shock regulon in E. coli [20, 27]. Interestingly, heat shock proteins GroS and GroL are required for proper folding of the major capsid proteins of coliphage PRD1 [37]. In several microorganisms, phage infection changed the expression of genes related to the stringent response, which leads to a shutoff of the bacterial protein synthesis machinery [20, 23, 25]. Poranen et al. [20] suggested that this phenomenon might be a consequence of nutrient limitation rather than a mechanism to boost viral protein synthesis, as protein synthesis shutoff was observed after production of the virion structural components. Several studies reported the induction of cell wall stress response genes in L. lactis upon phage infection [21, 31]. In $Y$. enterocolitica, infection by phage fR1-37 led to the upregulation of genes involved in phage-, cold- and osmotic shock [38]. Clearly, undergoing the lytic cycle is a stressor for the cell. However, we are yet to know if uninfected cells also display a response to predation. difference between life and death. Indeed, planktonic cells may eventually be eradicated by the virus (c) whereas the biofilm lifestyle may delay phage propagation thanks to the matrix and the lower metabolic rate of cells in deep layers of the biofilm (d). Moreover, there are examples of increased eDNA or polysaccharide production in response to viral predation (d) thereby enhancing biofilm formation and/or stability

\section{Impact of bacteriophages on virulence, antibiotic resistance, and biofilm formation}

\section{Virulence and antibiotic resistance}

Besides spreading virulence and antibiotic resistance markers among bacteria, bacteriophages can promote the expression of virulence/resistance traits of the infected cell. For instance, a polylysogenic Enterococcus faecalis strain displayed greater virulence than the prophage-free isogenic strain in sepsis and endocarditis animal models [39]. Similarly, temperate phage Pf4 participates in the pathogenicity of its host, $P$. aeruginosa, as shown in a mouse infection model [40]. Lysogeny also enabled long-term colonization of the redworm intestinal tract by Bacillus anthracis, and enhanced fitness of $P$. aeruginosa in a chronic lung infection model [41, 42]. Veses-Garcia et al. [29] observed that the presence of Shiga toxigenic prophages increased acid resistance in $E$. coli and, as a result, helped pathogenic strains to survive in the acidic environment of the stomach. Additionally, in some cases, phages confer greater resistance to antibiotics. Thus, presence of the gamma phage in $B$. anthracis and cryptic prophages in 
E. coli increases resistance to fosfomycin and ciprofloxacin/ $\beta$-lactams, respectively $[43,44]$. All these examples, however, correspond to prophages because there is practically no research regarding the effect of lytic predation on the virulence and antibiotic resistance of bacteria. This phenomenon is more difficult to study as the phage to bacteria ratios must be tightly controlled to avoid lysis of the entire bacterial population. Nonetheless, such experiments would be important to determine how virulent bacteriophages affect these phenotypes in natural communities. Moreover, this phenomenon has implications for the use of phage therapy in the clinic.

\section{Biofilm formation}

In the environment, bacterial cells are commonly organized in multicellular sessile communities called biofilms. Bacteria-phage interactions in biofilms are complex and still not fully understood (Fig. 2). Some authors believe that biofilms offer spatial refuges to phage-sensitive bacterial cells, perhaps due to the particular metabolic state of biofilm cells [45]. In contrast, Abedon [46] proposed that biofilm protection would only be effective under low phage pressure. Also, this protective effect would be limited to mature biofilm targets, in which phage propagation would be delayed [47, 48].

There is growing evidence that bacteriophages can modulate biofilm development (Table 1). Most data available to date reflects the participation of prophages on biofilm formation. Indeed, lysogens frequently form biofilms more readily than their non-lysogenic counterparts. For instance, Shewanella oneidensis MR-1 requires the presence of three prophages to release extracellular DNA, an essential component of the biofilm matrix [49]. This irondependent process is regulated by RecA [50]. However, prophage carriage does not always lead to enhanced biofilm formation. For example, addition of the QS molecule AI-2 or the antibiotic ciprofloxacin-triggered biofilm dispersal in E. faecalis via prophage induction [39]. Also, excision of the E. coli prophage $r a c$ is induced by the RpoS sigma factor during biofilm formation, ultimately leading to biofilm dispersal [51]. In P. aeruginosa PA14, phage DMS3 leads to lesser biofilm development through a mechanism dependent on the CRISPR-Cas system of the host [52]. Conversely, the filamentous prophage Pf4 participates in different stages of biofilm formation in P. aeruginosa [40]. The strain-lacking Pf4, for example, formed small and unstable microcolonies that did not exhibit some typical features such as the accumulation of dead cells and subsequent formation of hollow centers.

Information regarding the influence of virulent phages on biofilm formation is more limited, and mostly relates to the study of biofilm eradication with high-phage titers.

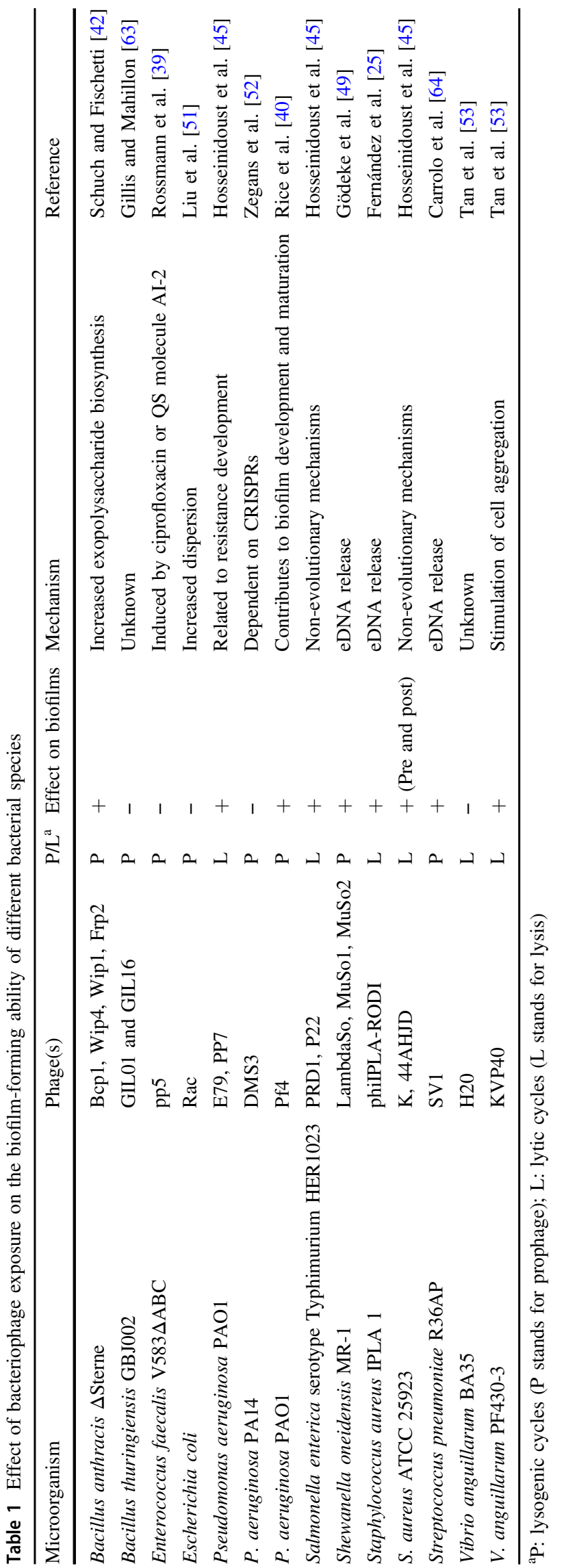


Fig. 3 Regulation of phage susceptibility by quorumsensing (QS) signaling. Bacterial cells control their antiphage strategy depending on cell density through QS signals. Thus, accumulation of QS molecules may trigger different strategies depending on the microorganism that will ultimately result in a greater ability of the bacterial population to withstand a phage attack

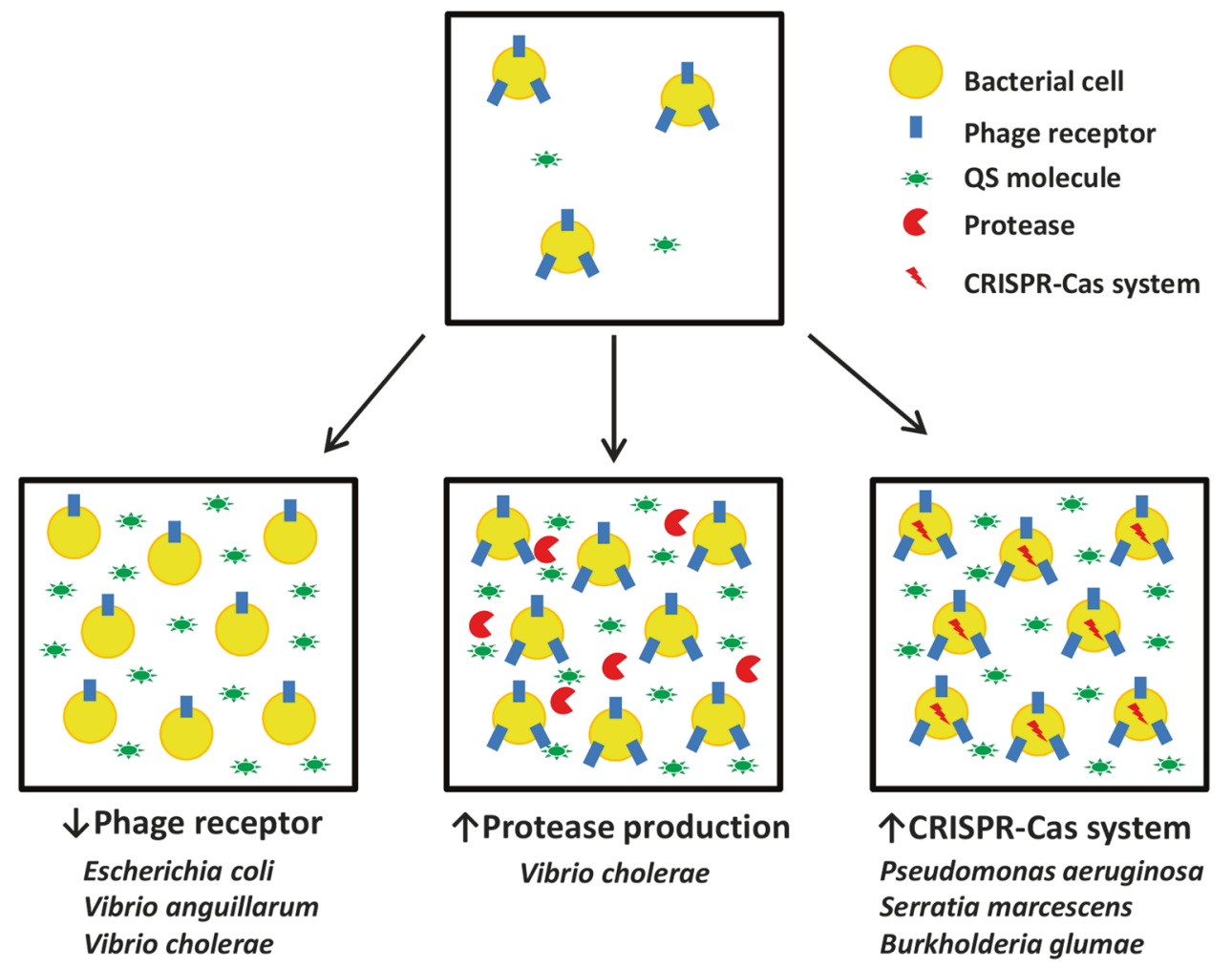

However, Hosseinidoust et al. [45] reported that the presence of species-specific phages promoted biofilm formation in three different pathogens. This increase involved the selection of phage-resistant cells with a strong ability to form biofilms in $P$. aeruginosa. In contrast, increased biofilm formation in $S$. aureus and $S$. enterica appeared to be linked to non-evolutionary mechanisms [45]. Another study showed that exposure of $V$. anguillarum PF430-3 to phage KVP40 promoted biofilm formation through increased cell aggregation [53]. More recently, Fernández et al. [25] reported that exposure of some $S$. aureus strains to lowlevel phage concentrations enhanced biofilm development due to accumulation of eDNA in the extracellular matrix. Consequently, low-phage pressure may in some cases favor conditions that protect the bacterial cells from external challenges, including attack by other phages or antimicrobial agents.

\section{Role of bacterial communication under phage pressure}

Despite being single-celled organisms, bacteria can communicate by using chemical signals that elicit physiological responses in neighboring cells, such as the well-known quorum-sensing (QS) systems. Although still not fully understood, the importance of cell-to-cell communication for the development of microbial communities is well documented. Additionally, QS signaling typically coordinates the expression of virulence determinants in pathogenic bacteria. In the last few years, different authors have established an interesting correlation between the production of QS molecules and phage susceptibility (Fig. 3). These findings emphasize the role of phages as an integral part of microbial communities, as their ability to infect their bacterial hosts is regulated by signals controlling population development. Several studies have shed light on the specific molecular mechanisms that coordinate QS and phage resistance. In some cases, increased production of QS signals leads to a lower expression of phage-receptorencoding genes, which lessens the adsorption rate. This, in turn, confers decreased susceptibility to viral infection. In Escherichia coli, for example, the presence of lambda receptors decreased in response to $\mathrm{N}$-acyl-L-homoserine lactone (AHL) production [54]. Similarly, Tan et al. [55] found that QS molecules regulated the levels of OmpK, the receptor of phage KVP40, on the surface of Vibrio anguillarum cells. In this pathogen, antiphage defenses are tightly regulated within the quorum-sensing circuit. Thus, protection from phage attack at low cell densities, when QS levels are low, is achieved by formation of cell aggregates. In Vibrio cholerae, QS enhances phage resistance by two different mechanisms, downregulation of a phage receptor (LPS-O antigen) and upregulation of the gene encoding a haemaglutinin protease shown to inactivate vibriophages [56]. There is also evidence that QS signals can modulate the expression of genes involved in the CRISPR-Cas 
systems of P. aeruginosa [57], Burkholderia glumae [58], and Serratia [59]. This immunity mechanism protects bacteria from virus infection, but can be costly to the cell and lead to reduced fitness. Therefore, the microorganism would benefit from the specific activation of CRISPR-Cas systems when the population is most susceptible to phage attack, which, according to the Kill the Winner hypothesis, occurs at high cell densities [60]. Interestingly, Hargreaves et al. [61] described that phiCDHM1, a bacteriophage that infects Clostridium diffficile, carried the genes necessary to produce a QS molecule. This would potentially allow the phage to control the development of the host population. Another remarkable discovery was the identification of a viral communication system, arbitrium, based on the synthesis of a small peptide whose accumulation favored lysogeny, thereby preventing extinction of the host population [62]. This new finding adds a further layer of complexity to the coordination of host-phage populations. Perhaps, future studies will determine if bacterial cells infected by a lytic phage produce some kind of molecule to "warn" neighboring cells that they need to protect themselves from phage attack.

\section{Concluding remarks}

Bacteriophages are generally perceived as the natural enemies of bacteria. However, an evolutionary analysis of phage-host dynamics suggests that predator and prey often co-evolve in such a way as to avoid complete eradication. Evidence of this trend can be observed in the intricate regulation of phage sensitivity depending on bacterial population density, as well as the complex interplay between phage and host, especially in biofilms. Overall, the available information hints that both temperate and virulent phages, while remaining a threat to individual cells, have primarily been allies of bacterial communities by enhancing their adaptation to the surrounding environment and modulating bacterial competition. Therefore, it is essential to understand phages to fully comprehend the biology of bacteria. Moreover, efforts made to use bacteriophages as antimicrobials should consider that these methods are designed to alter the equilibrium between host and predator populations, moving this equilibrium towards host eradication. It is, therefore, paramount to avoid undesired effects on microbial ecosystems. After all, bacteriophages are both friends and foes of microbes depending on the context. If we understand the principles that govern this relationship, we may be able to tame bacteriophages for our benefit without negatively affecting their natural balance in the environment.

Acknowledgments Our work on bacteriophages was funded by grants AGL2015-65673-R (Ministry of Science and Innovation, Spain), EU
ANIWHA ERA-NET BLAAT, GRUPIN14-139 (Program of Science, Technology and Innovation 2013-2017 and FEDER EU funds, Principado de Asturias, Spain). L.F. was awarded a "Marie Curie ClarinCofund" grant. P.G. and A.R. are members of the bacteriophage network FAGOMAII and the FWO Vlaanderen funded "Phagebiotics" research community (WO.016.14).

\section{Compliance with ethical standards}

Conflict of interest The authors declare that they have no conflict of interest.

\section{References}

1. d'Herelle F. Sur un microbe invisible antagoniste des bacilles dysentériques. CR Acad Sci. 1917;165:373-75.

2. Twort FW. An investigation on the nature of ultra-microscopic viruses. Lancet. 1915; ii:1241-43.

3. Abedon ST, García P, Mullany P, Aminov R. Editorial: phage therapy: past, present and future. Front Microbiol. 2017;8:981.

4. Clokie MR, Millard AD, Letarov AV, Heaphy S. Phages in nature. Bacteriophage. 2011;1:31-45.

5. Rascovan N, Duraisamy R, Desnues C. Metagenomics and the human virome in asymptomatic individuals. Annu Rev Microbiol. 2016;70:125-41.

6. Salmond GP, Fineran PC. A century of the phage: past, present and future. Nat Rev Microbiol. 2015;13:777-86.

7. Barrangou R, Fremaux C, Deveau H, Richards M, Boyaval P, Moineau $S$, et al. CRISPR provides acquired resistance against viruses in prokaryotes. Science. 2007;315:1709-12.

8. Jinek M, Chylinski K, Fonfara I, Hauer M, Doudna JA, Charpentier E. A programmable dual-RNA-guided DNA endonuclease in adaptive bacterial immunity. Science. 2012;337:816-21.

9. Casjens S. Prophages and bacterial genomics: what have we learned so far? Mol Microbiol. 2003;49:277-300.

10. Filippov AA, Sergueev KV, He Y, Huang XZ, Gnade BT, Mueller $\mathrm{AJ}$, et al. Bacteriophage-resistant mutants in Yersinia pestis: identification of phage receptors and attenuation for mice. PLoS ONE. 2011;6:e25486.

11. Scott AE, Timms AR, Connerton PL, Loc Carrillo C, Adzfa Radzum K, Connerton IF. Genome dynamics of Campylobacter jejuni in response to bacteriophage predation. PLoS Pathog. 2007;3:e119.

12. Hosseinidoust Z, van de Ven TG, Tufenkji N. Evolution of Pseudomonas aeruginosa virulence as a result of phage predation. Appl Environ Microbiol. 2013;79:6110-16.

13. Davies EV, James CE, Williams D, O'Brien S, Fothergill JL, Haldenby $S$, et al. Temperate phages both mediate and drive adaptive evolution in pathogen biofilms. Proc Natl Acad Sci USA. 2016;113:8266-71.

14. Cairns J, Frickel J, Jalasvuori M, Hiltunen T, Becks L. Genomic evolution of bacterial populations under coselection by antibiotics and phage. Mol Ecol. 2016;26:1848-59.

15. Koskella B, Brockhurst MA. Bacteria-phage coevolution as a driver of ecological and evolutionary processes in microbial communities. FEMS Microbiol Rev. 2014;38:916-31.

16. Fortier LC, Sekulovic O. Importance of prophages to evolution and virulence of bacterial pathogens. Virulence. 2013;4:354-65.

17. Haaber J, Leisner JJ, Cohn MT, Catalan-Moreno A, Nielsen JB, Westh $\mathrm{H}$, et al. Bacterial viruses enable their host to acquire antibiotic resistance genes from neighbouring cells. Nat Commun. 2016;7:13333. 
18. Modi SR, Lee HH, Spina CS, Collins JJ. Antibiotic treatment expands the resistance reservoir and ecological network of the phage metagenome. Nature. 2013;499:219-22.

19. Keen EC, Bliskovsky VV, Malagon F, Baker JD, Prince JS, Klaus JS, et al. Novel "superspreader" bacteriophages promote horizontal gene transfer by transformation. mBio. 2017;8:e2115-16.

20. Poranen MM, Ravantti JJ, Grahn AM, Gupta R, Auvinen P, Bamford DH. Global changes in cellular gene expression during bacteriophage PRD1 infection. J Virol. 2006;80:8081-88.

21. Ainsworth S, Zomer A, Mahony J, van Sinderen D. Lytic infection of Lactococcus lactis by bacteriophages Tuc2009 and c2 triggers alternative transcriptional host responses. Appl Environ Microbiol. 2013;79:4786-98.

22. Doron S, Fedida A, Hernández-Prieto MA, Sabehi G, Karunker I, Stazic D, et al. Transcriptome dynamics of a broad host-range cyanophage and its hosts. ISME J. 2016;10:1437-55.

23. De Smet J, Zimmermann M, Kogadeeva M, Ceyssens PJ, Vermaelen W, Blasdel B, et al. High coverage metabolomics analysis reveals phage-specific alterations to Pseudomonas aeruginosa physiology during infection. ISME J. 2016;10:1823-35.

24. Blasdel BG, Chevallereau A, Monot M, Lavigne R, Debarbieux L. Comparative transcriptomics analyses reveal the conservation of an ancestral infectious strategy in two bacteriophage genera. ISME J. 2017;11:1988-96.

25. Fernández L, González S, Campelo AB, Martínez B, Rodríguez A, García P. Low-level predation by lytic phage phiIPLA-RODI promotes biofilm formation and triggers the stringent response in Staphylococcus aureus. Sci Rep. 2017;7:40965.

26. Lavigne R, Lecoutere E, Wagemans J, Cenens W, Aertsen A, Schoofs L, et al. A multifaceted study of Pseudomonas aeruginosa shutdown by virulent podovirus LUZ19. mBio. 2013;24: e00061-13.

27. Osterhout RE, Figueroa IA, Keasling JD, Arkin AP. Global analysis of host response to induction of a latent bacteriophage. BMC Microbiol. 2007;7:82.

28. Ravantti JJ, Ruokoranta TM, Alapuranen AM, Bamford DH. Global transcriptional responses of Pseudomonas aeruginosa to phage PRR1 infection. J Virol. 2008;82:2324-29.

29. Veses-Garcia M, Liu X, Rigden DJ, Kenny JG, McCarthy AJ, Allison HE. Transcriptomic analysis of Shiga-toxigenic bacteriophage carriage reveals a profound regulatory effect on acid resistance in Escherichia coli. Appl Environ Microbiol. 2015;81:8118-25.

30. Zhao X, Chen C, Shen W, Huang G, Le S, Lu S, et al. Global transcriptomic analysis of interactions between Pseudomonas aeruginosa and bacteriophage PaP3. Sci Rep. 2016;6:19237.

31. Fallico V, Ross RP, Fitzgerald GF, McAuliffe O. Genetic response to bacteriophage infection in Lactococcus lactis reveals a four-strand approach involving induction of membrane stress proteins, d-alanylation of the cell wall, maintenance of proton motive force, and energy conservation. J Virol. 2011;85:12032-42.

32. Dendooven T, Van den Bossche A, Hendrix H, Ceyssens PJ, Voet $\mathrm{M}$, Bandyra KJ, et al. Viral interference of the bacterial RNA metabolism machinery. RNA Biol. 2017;14:6-10.

33. Van den Bossche A, Hardwick SW, Ceyssens PJ, Hendrix H, Voet M, Dendooven T, et al. Structural elucidation of a novel mechanism for the bacteriophage-based inhibition of the RNA degradosome. Elife. 2016;5:e16413.

34. Chevallereau A, Blasdel BG, De Smet J, Monot M, Zimmermann M, Kogadeeva M, et al. Next-Generation "-omics" approaches reveal a massive alteration of host RNA metabolism during bacteriophage infection of Pseudomonas aeruginosa. PLoS Genet. 2016;12:e1006134.

35. Aranda M, Maule A. Virus-induced host gene shutoff in animals and plants. Virology. 1998;243:261-67.
36. Campoy S, Hervàs A, Busquets N, Erill I, Teixidó L, Barbé J. Induction of the SOS response by bacteriophage lytic development in Salmonella enterica. Virology. 2006;351:360-67.

37. Hänninen $\mathrm{AL}$, Bamford $\mathrm{DH}$, Bamford JK. Assembly of membrane-containing bacteriophage PRD1 is dependent on GroEL and GroES. Virology. 1997;227:207-10.

38. Leskinen K, Blasdel BG, Lavigne R, Skurnik M. RNAsequencing reveals the progression of phage-host interactions between fR1-37 and Yersinia enterocolitica. Viruses. 2016;8:111.

39. Rossmann FS, Racek T, Wobser D, Puchalka J, Rabener EM, Reiger M, et al. Phage-mediated dispersal of biofilm and distribution of bacterial virulence genes is induced by quorum sensing. PLoS Pathog. 2015;11:e1004653.

40. Rice SA, Tan CH, Mikkelsen PJ, Kung V, Woo J, Tay M, et al. The biofilm life cycle and virulence of Pseudomonas aeruginosa are dependent on a filamentous prophage. ISME J. 2009;3:271-82.

41. Davies EV, James CE, Kukavica-Ibrulj I, Levesque RC, Brockhurst MA, Winstanley C. Temperate phages enhance pathogen fitness in chronic lung infection. ISME J. 2016;10:2553-55.

42. Schuch R, Fischetti VA. The secret life of the anthrax agent Bacillus anthracis: bacteriophage-mediated ecological adaptations. PLoS ONE. 2009; 4:e6532.

43. Schuch R, Fischetti VA. Detailed genomic analysis of the Wbeta and gamma phages infecting Bacillus anthracis: implications for evolution of environmental fitness and antibiotic resistance. J Bacteriol. 2006;188:3037-51.

44. Wang X, Kim Y, Ma Q, Hong SH, Pokusaeva K, Sturino JM, et al. Cryptic prophages help bacteria cope with adverse environments. Nat Commun. 2010;1:147.

45. Hosseinidoust Z, Tufenkji N, van de Ven TG. Formation of biofilms under phage predation: considerations concerning a biofilm increase. Biofouling. 2013a;29:457-68.

46. Abedon ST. Spatial vulnerability: bacterial arrangements, microcolonies, and biofilms as responses to low rather than high phage densities. Viruses. 2012;4:663-87.

47. Abedon ST. Bacteriophage exploitation of bacterial biofilms: phage preference for less mature targets? FEMS Microbiol Lett. 2016;363:fnv246.

48. Abedon ST. Phage "delay" towards enhancing bacterial escape from biofilms: a more comprehensive way of viewing resistance to bacteriophages. AIMS Microbiol. 2017;3:186-26.

49. Gödeke J, Paul K, Lassak J, Thormann KM. Phage-induced lysis enhances biofilm formation in Shewanella oneidensis MR-1. ISME J. 2011;5:613-26.

50. Binnenkade L, Teichmann L, Thormann KM. Iron triggers $\lambda$ So prophage induction and release of extracellular DNA in Shewanella oneidensis MR-1 biofilms. Appl Environ Microbiol. 2014;80:5304-16.

51. Liu X, Li Y, Guo Y, Zeng Z, Li B, Wood TK, et al. Physiological function of rac prophage during biofilm formation and regulation of rac excision in Escherichia coli K-12. Sci Rep. 2015;5:16074.

52. Zegans ME, Wagner JC, Cady KC, Murphy DM, Hammond JH, O'Toole GA. Interaction between bacteriophage DMS3 and host CRISPR region inhibits group behaviors of Pseudomonas aeruginosa. J Bacteriol. 2009;191:210-19.

53. Tan D, Dahl A, Middelboe M. Vibriophages differentially influence biofilm formation by Vibrio anguillarum strains. Appl Environ Microbiol. 2015;81:4489-97.

54. Høyland-Kroghsbo NM, Maerkedahl RB, Svenningsen SL. A quorum-sensing-induced bacteriophage defense mechanism. mBio. 2013;4:e00362-12.

55. Tan D, Svenningsen SL, Middelboe M. Quorum sensing determines the choice of antiphage defense strategy in Vibrio anguillarum. mBio. 2015;6:e00627. 
56. Hoque MM, Naser IB, Bari SM, Zhu J, Mekalanos JJ, Faruque SM. Quorum regulated resistance of Vibrio cholerae against environmental bacteriophages. Sci Rep. 2016;6:37956.

57. Høyland-Kroghsbo NM, Paczkowski J, Mukherjee S, Broniewski J, Westra E, Bondy-Denomy J, et al. Quorum sensing controls the Pseudomonas aeruginosa CRISPR-Cas adaptive immune system. Proc Natl Acad Sci USA. 2017;114:131-35.

58. Gao R, Krysciak D, Petersen K, Utpatel C, Knapp A, Schmeisser $\mathrm{C}$, et al. Genome-wide RNA sequencing analysis of quorum sensing-controlled regulons in the plant-associated Burkholderia glumae PG1 strain. Appl Environ Microbiol. 2015;81:7993-8007.

59. Patterson AG, Jackson SA, Taylor C, Evans GB, Salmond GP, Przybilski R, et al. Quorum sensing controls adaptive immunity through the regulation of multiple CRISPR-Cas systems. Mol Cell. 2016;64:1102-8.

60. Winter C, Bouvier T, Weinbauer MG, Thingstad TF. Trade-offs between competition and defense specialists among unicellular planktonic organisms: the "killing the winner" hypothesis revisited. Microbiol Mol Biol Rev. 2010;74:42-57.

61. Hargreaves KR, Kropinski AM, Clokie MRJ. What does the talking? Quorum sensing signalling genes discovered in a bacteriophage genome. PLoS ONE. 2014;9:e85131.

62. Erez Z, Steinberger-Levy I, Shamir M, Doron S, Stokar-Avihail A, Peleg Y, et al. Communication between viruses guides lysislysogeny decisions. Nature. 2017;541:488-93.

63. Gillis A, Mahillon J. Influence of lysogeny of Tectiviruses GIL01 and GIL16 on Bacillus thuringiensis growth, biofilm formation, and swarming motility. Appl Environ Microbiol. 2014;80:7620-30.

64. Carrolo M, Frias MJ, Pinto FR, Melo-Cristino J, Ramirez M. Prophage spontaneous activation promotes DNA release enhancing biofilm formation in Streptococcus pneumoniae. PLoS ONE. 2010;5:e15678. 